\title{
Analysis on the Transfer Characteristics of Rogowski-coil Current Transformer and Its Influence on Protective Relaying
}

\author{
Yi Wang ${ }^{1}$, Jiaman $\mathrm{Li}^{2}$, Yulan $\mathrm{Hu}^{1}$, Ranran $\mathrm{An}^{1}$, Zexiang Cai ${ }^{2}$, Ruiwen $\mathrm{He}^{3}$ \\ ${ }^{1}$ Electric Power Research Institute, Guangdong Power Grid Corporation, Guangzhou, China \\ ${ }^{2}$ School of Electric Power, South China University of Technology, Guangzhou, China \\ ${ }^{3}$ School of Automation, Guangdong University of Technology, Guangzhou, China \\ Email:mr-wangyi@163.com, li.jiaman@mail.scut.edu.cn, anranran85@163.com,epzxcai@scut.edu.cn
}

Received March, 2013

\begin{abstract}
This paper systematically analyzes the transfer characteristics of the Rogowski-coil Current Transformer and its effect on protective relaying through theoretical analysis, experiments and simulations. The frequency characteristics and transient characteristics of Rogowski transducer and Rogowski-coil Current Transformer are deeply analyzed based on the physical structure of the transformer. It is revealed that broad bandwidth of the transformer can improve the performance of protective relaying, and the bandwidth is determined mainly by the parameters of the Rogowski transducer and signal processing circuits. It is also discovered that the measurement errors of transient current mainly depend on the abilities for the current transformer to reproduce an accurate replica of the decaying dc components, which is mainly decided by the decay time constant of the aperiodic component of transient current and the parameters of the integral unit. Finally, some measures are proposed for the performance improvement of Rogowski-coil Current Transformer to meet the requirements of protective relaying system in terms of structural design and testing standards.
\end{abstract}

Keywords: Current Transformer; Rogowski-coil; Transfer Characteristics; Protective Relaying

\section{Introduction}

The Rogowski-coil Current Transformer has attracted much attention and expectation as it can meet the requirements of power system protective relaying for its excellent performances, including a good linearity for measurement, broad bandwidth, excellent dynamic response and no magnetic saturation etc. [1,2]

Scholars have done a lot of research on the Rogowskicoil current transformer about its sensors [3-6], power supply [7], electronic circuit [8], integral method [9] and merging unit [10], which have effectively pushed forward the Rogowski-coil current transformer to be practical. Reference [11] discussed the calculation method and measurement principle of distributed capacitance and its effects on dynamic characteristics of sensors in time domain and frequency domain. The integral time constant is the major factor affecting the response of transformer to the DC components of transient current [12]. In order to improve the performance of the transformers, PCB structure and T-integrator were adopted when measuring the transient current in power system in reference [13]. Simulation analysis and experiments on the frequency characteristics, steady-state performance and transient performance of PCB transformer have been done in reference [14]. These studies show that Rogowski-coil Current Transformer has a good steady-state and dynamic performance for measurement in terms of structural design, but they do not point out its problems on transfer characteristics from the aspect of application.

Rogowski-coil current transformer has a linear characteristic, which means the superposition principle can be applied. Thus the transfer characteristic of Rogowskicoil current transformer can be quantitatively analyzed, which is quite different from the electromagnetic current transformers. The outputs of the electromagnetic current transformers have uncertainty due to its nonlinear magnetic characteristics. The testing and evaluation standards $[15,16]$ of electronic current transformer have failed to reflect the structural and transfer characteristics of Rogowski-coil Current Transformer.

So far the application of Rogowski-coil Current Transformer is subjected to its stability and reliability. Besides this, the lack of research on transfer characteristics and the inadequate understanding of the existing problems, as well as the overgeneralizations of the relevant test standards, have seriously affected the maturity and extensive 
application of this new equipment. The protective relaying system has to adapt to the characteristics of electronic transformer in the practical application $[17,18]$ in this situation.

From the perspective of protective relaying system application, this paper deeply studies the frequency characteristics and transient response of the Rogowski transducer and the Rogowski-coil current transformer, combined with experimental testing and simulation results, analyzes the key factors affecting transfer characteristics of the transformer, and offers the proposals for Rogowskicoil Current Transformer to adapt to the needs of protective relaying.

\section{Transfer Characteristics of Rogowski Transducer}

The structure of Rogowski-coil Current Transformer can be divided into Rogowski transducer, analog signal processing unit, digital signal processing and transmission units, merging unit and power supply unit according to its function.

The equivalent circuit [12] of the Rogowski transducer is shown in Figure 1 Rogowski transducer has a fairly small mutual inductance due to its air core. The Rogowskicoil Current Transformer dedicated to protective relaying works in the outside integral way, and the load resistance is very large. When the measured current has a low frequency, and coil internal resistance and interturn capacitance is tiny enough to be ignored, secondary side of the Rogowski transducer is close to be in open condition. At the moment, the output voltage is

$$
u_{1}(t) \approx e(t)=-M \cdot \mathrm{d} i / \mathrm{d} t .
$$

\subsection{Frequency Characteristic}

The transfer function for Rogowski transducer according to the equivalent circuit is

$$
\mathrm{H}_{1}(\mathrm{~s})=\frac{U_{1}(s)}{I(s)}=\frac{M s}{L_{0} C_{0} s^{2}+\left(\frac{L_{0}}{\mathrm{R}_{L}}+\mathrm{R}_{0} \mathrm{C}_{0}\right) s+\left(\frac{R_{0}}{\mathrm{R}_{L}}+1\right)}
$$

The undamped natural angular frequency

$$
\omega_{n}=\frac{1}{\sqrt{L_{0} C_{0}}} \sqrt{\frac{R_{L}+R_{0}}{R_{L}}},
$$

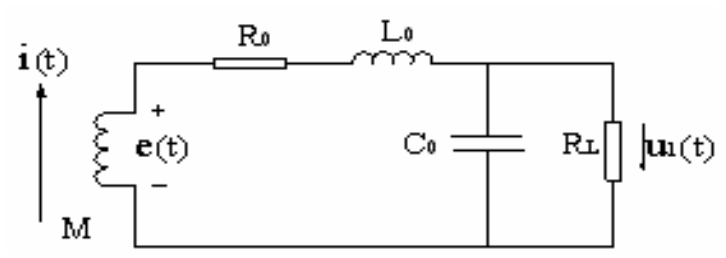

Figure 1. Equivalent circuit of Rogowski transducer. damping ratio

$$
\zeta=\frac{1}{2 \sqrt{L_{0} C_{0}}}\left(\frac{L_{0}}{R_{L}}+R_{0} C_{0}\right) \sqrt{\frac{R_{L}}{R_{L}+R_{0}}},
$$

and then the transfer function is

$$
H_{1}(s)=\frac{M}{L_{0} C_{0}} \cdot \frac{s}{\left(s-s_{1}\right) \cdot\left(s-s_{2}\right)}
$$

Where characteristic roots are $s_{1,2}=-\zeta \omega_{n} \pm \omega_{n} \sqrt{\zeta^{2}-1}$.

When primary current is the rated current $I_{N}$, the induction potential of Rogowski transducer is $E=$ $\omega M I_{N}$, therefore, the manufacturers determine the number of turns of coil $N$ depending on the desired value of the mutual inductance $M$. And the coil induction is is $L_{0}=M \cdot N$. Then the main factors affecting the frequency characteristics of Rogowski transducer are the internal resistance of the coil and distributed capacitance.

The Bode diagram of the transfer function of Rogowski transducer is shown in Figure 2. Phase error increases as the frequency increases. When the internal resistance and the distributed capacitance vary individually with other parameters remaining unchanged, the Bode diagrams of the Rogowski transducer are shown in Figures 2(a) and (b), from which we can know the impacts of the internal resistance and distributed capacitance on the bandwidth of Rogowski transducer should not be ignored.

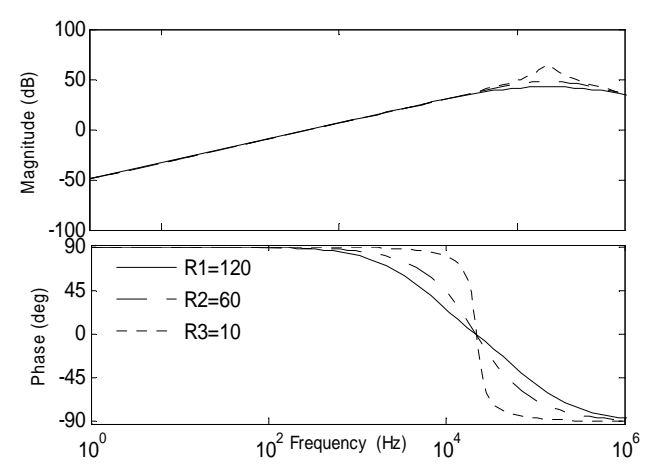

(a) characteristics when Internal resistance changes

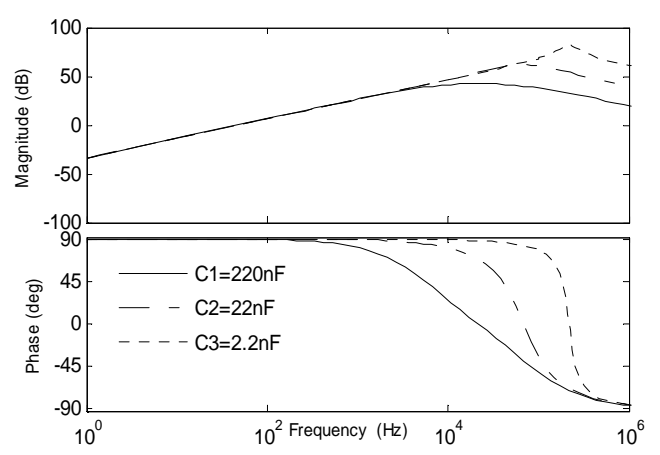

(b) characteristics when distributed capacitance changes

Figure 2. Bode diagram of Rogowski transducer. 
To reduce the distributed capacitance can significantly increase the upper cutoff frequency. Changing the resistance will change the damping ratio, so that the frequency characteristics can be changed. When designing Rogowski transducer, select a suitable wire diameter and minimize the distributed capacitance under the promise of appropriately selecting the coil structure parameters, so as to improve the high-frequency characteristics of the Rogowski transducer.

\subsection{Transient Characteristics}

The Rogowski-coil Current Transformer has a good linearity for measurement, no saturation problem, so the transient response of Rogowski-coil Current Transformer can be analyzed using superposition principle, and the response of the transformer to each component of transient current can be studied respectively. In other words, the transient analysis of Rogowski-coil Current Transformer can focus on studying the effects of decaying aperiodic component on transformers based on frequency response analysis.

When the primary current is the aperiodic component decaying with the time constants $\tau, i(t)=I_{m} e^{-t / \tau}$,

The corresponding Laplace transform is

$$
I(s)=\frac{I_{m}}{s+1 / \tau},
$$

then the sensor output is given by

$$
\mathrm{U}_{1}(s)=\frac{M}{L_{0} C_{0}} \times \frac{s}{\left(s-s_{1}\right) \times\left(s-s_{2}\right)} \times \frac{I_{m}}{s+1 / t}
$$

Transform into the partial fraction

$$
U_{1}(\mathrm{~s})=\frac{M I_{m}}{L_{0} C_{0}} \cdot\left(\frac{A_{1}}{s-s_{1}}+\frac{B_{1}}{s-s_{2}}+\frac{C_{1}}{s+1 / \tau}\right)
$$

where $\quad A_{1}=\frac{s_{1}}{\left(s_{1}-s_{2}\right)\left(s_{1}+1 / \tau\right)}, \quad B_{1}=\frac{s_{2}}{\left(s_{2}-s_{1}\right)\left(s_{2}+1 / \tau\right)}$,

$$
C_{1}=\frac{-\tau}{\left(1+\tau s_{1}\right)\left(1+\tau s_{2}\right)} \text {. }
$$

By inverse Laplace transform, time domain expression of sensor output is

$$
u_{1}(t)=\frac{M I_{m}}{L_{0} C_{0}}\left(A_{1} e^{s_{1} t}+B_{1} e^{s_{2} t}+C_{1} e^{-t / \tau}\right)
$$

In the underdamping situation $\zeta<1, s_{1}$ and $s_{2}$ are a pair of complex conjugate roots, and the decay rates of the first two items depend on the absolute values of the characteristic roots' real parts, when in overdamping situation $\zeta>1, s_{1}$ and $s_{2}$ are two negative real roots, the decay rates of the first two items depend on $\left(\zeta \pm \sqrt{\zeta^{2}-1}\right) \omega_{n}$. As the distributed capacitance of the sensor is very small, then $\omega_{n}$ is generally above $10^{5}$, the first two items decay very quickly regardless of the damping, with negligible impact on the output voltage. For $s_{1}+s_{2}=-2 \zeta \omega_{n}, s_{1} \cdot s_{2}=\omega_{n}^{2}$, the output voltage corresponding to input decaying DC component is given by

$$
u_{1}(t)=M I_{m} \frac{-\tau}{L_{0} C_{0}-\tau \cdot R_{0} C_{0}+\tau^{2}} e^{-t / \tau}
$$

$L_{0} C_{0}$ and $R_{0} C_{0}$ are very small compared to $\tau$, so the equation above is approximately

$$
u_{1}(t)=M I_{m} \frac{1}{-\tau} e^{-t / \tau}
$$

Simulation shows that after $0.5 \mathrm{~ms}$, the output of the equation (7) is exactly the same with that of equation (5), which illustrates the simple differential model, can describe the response of sensor to the decaying aperiodic component. The error of the Rogowski-coil Current Transformer when transforming the a periodic component mainly depends on the following integral circuit whether can accurately restore the differential signal into decaying DC or not.

\section{The Transfer Characteristics of Rogows- ki-coil Current Transformer}

The integral unit in the prototype discussed in this paper is implemented by analog circuit. The $\mathrm{A} / \mathrm{D}$ conversion and the following link in the signal acquisition system of transformers can only change the signal form or its carrier, although in which the inevitable errors also exit, they does not change the signal transfer characteristic. So this paper only discusses the model and characteristic of Rogowski transducer and analog signal processing circuits. In order to react Frequency characteristics more realistically, leave out the low-pass filter in the signal processing circuit.

\subsection{Frequency Characteristic}

The overall transfer function of Rogowski-coil Current Transformer is:

$$
H(s)=H_{1}(s) H_{2}(s) H_{3}(s)
$$

$H_{1}(s), H_{2}(s), H_{3}(s)$ is respectively the transfer function of Rogowski transducer, the integral amplification and phase compensation part .

$$
H_{2}(s)=\frac{K_{2}}{1+R C s}, \quad H_{3}(s)=\frac{1-K_{3} s}{1+K_{3} s}
$$

where $R C=0.066, K_{2}=19.56$. Based on the prototype parameters, the Bode diagram of the transformer as a whole is as showed in Figure $\mathbf{3}$ below it can be seen that the ratio error and phase error of the Rogowski-coil Current Transformer is smaller between 10-200 Hz. The phase error is larger in high frequency range. 


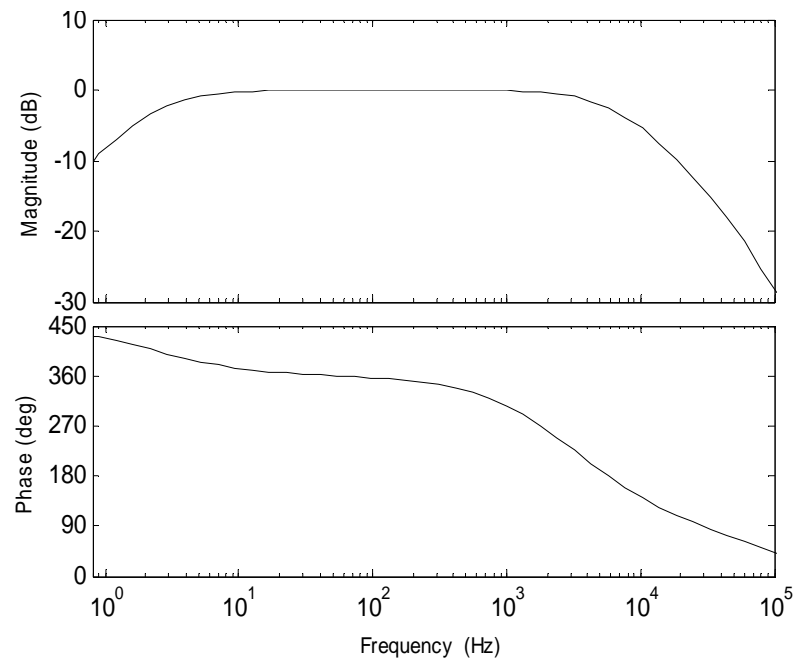

Figure 3. Bode diagram of Rogowski-coil Current Transformer.

\subsection{Transient Characteristics}

Similarly, set $I(s)=\frac{I_{m}}{s+1 / \tau}$, then the transformer output is

$$
U(s)=H(s) \frac{I_{m}}{s+1 / \tau}
$$

Rewrite it by

$$
U(\mathrm{~s})=\frac{A}{s-s_{1}}+\frac{B}{s-s_{2}}+\frac{C}{s+1 / R C}+\frac{D}{s+1 / K_{3}}+\frac{E}{s+1 / \tau}
$$

where

$$
\begin{aligned}
A= & \frac{M I_{m} K_{2}}{L_{0} C_{0} R C} \cdot \frac{s_{1}}{\left(s_{1}-s_{2}\right)} \cdot \frac{1}{s_{1}+1 / R C} \cdot \frac{1 / K_{3}-s_{1}}{1 / K_{3}+s_{1}} \cdot \frac{1}{s_{1}+1 / \tau} \\
B= & \frac{M I_{m} K_{2}}{L_{0} C_{0} R C} \cdot \frac{s_{2}}{\left(s_{2}-s_{1}\right)} \cdot \frac{1}{s_{2}+1 / R C} \cdot \frac{1 / K_{3}-s_{2}}{1 / K_{3}+s_{2}} \cdot \frac{1}{s_{2}+1 / \tau} \\
C= & \frac{M I_{m} K_{2}}{L_{0} C_{0} R C} \cdot \frac{-1 / R C}{\left(1 / R C+s_{1}\right) \cdot\left(1 / R C+s_{2}\right)} \cdot \frac{1 / K_{3}+1 / R C}{1 / K_{3}-1 / R C} \\
& \cdot \frac{1}{-1 / R C+1 / \tau} \\
D= & \frac{M I_{m} K_{2}}{L_{0} C_{0} R C} \cdot \frac{-2 / K_{3}^{2}}{\left(1 / K_{3}+s_{1}\right)\left(1 / K_{3}+s_{2}\right)} \cdot \frac{1}{-1 / K_{3}+1 / R C} \\
& \cdot \frac{1}{-1 / K_{3}+1 / \tau} \\
E= & \frac{M I_{m} K_{2}}{L_{0} C_{0} R C} \cdot \frac{-1 / \tau}{\left(1 / \tau+s_{1}\right) \cdot\left(1 / \tau+s_{2}\right)} \cdot \frac{1}{-1 / \tau+1 / R C} \cdot \frac{1 / K_{3}+1 / \tau}{1 / K_{3}-1 / \tau}
\end{aligned}
$$

As $s_{1}$ and $s_{2}$ are very large, $K_{3}$ is very small, the three corresponding items in expression (10) decay rapidly and so can be ignored. After simplification according to the features of $s_{1}, s_{2}$ and $K_{3}$, the transformer output voltage

$$
u(t)=\frac{M I_{m} K_{2}}{R C-\tau}\left(e^{-t / \tau}-\frac{\tau}{R C} e^{-t / R C}\right)
$$

Thus it can be seen that the response of Rogowski-coil Current Transformer to the decaying a periodic component is mainly associated with decay time constants and integral time constant. Equation (11) equals to the output results of the sensor using differential model with the integrator.

As shown in Figure 4, the maximum instantaneous error increases as the decay time constant increases. Remain the dc decay time constant at $80 \mathrm{~ms}$, the relations between maximum instantaneous error current and integral time constant is as Figure $\mathbf{5}$ shows, the error is smaller when the integral time constant $R C$ is between $0.05-0.1$. It can be found that the maximum instantaneous error at least above $30 \%$ in all situations. The experiment and simulation analysis both indicate that it is difficult for the simple RC integrator to satisfy the requirements of protective relaying on transient characteristics of transformer, namely the maximum peak instantaneous of Electronic transformer for transient protection cannot exceed $10 \%$. It is necessary to improve the performance of Rogowski-coil Current Transformer transferring aperiodic components by improving the structure of the integrator.

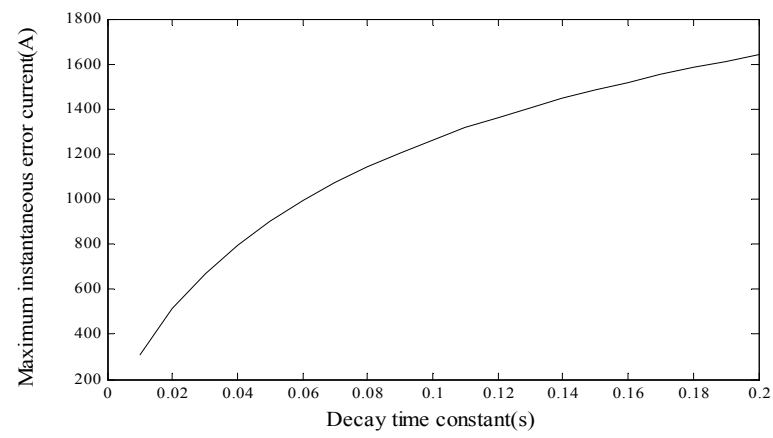

Figure 4. Relationship between the maximum instantaneous error current and the DC decay time constant.

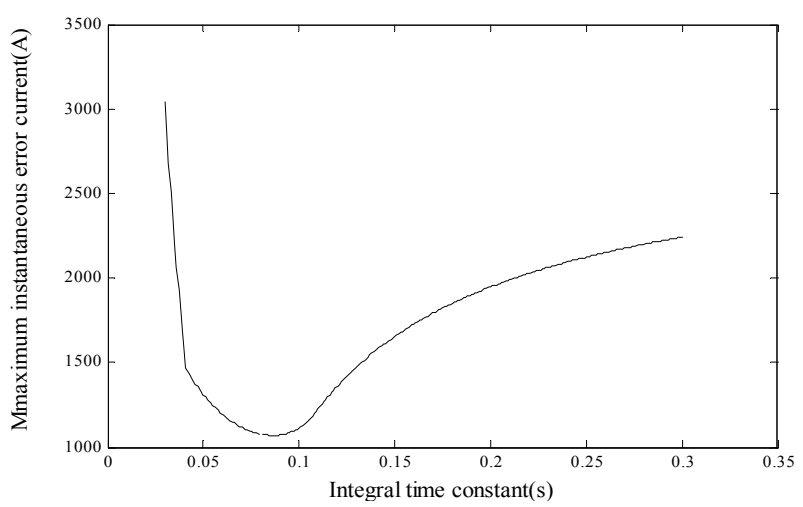

Figure 5. Relationship between the maximum instantaneous error current and the integral time constant. 


\section{Adaptability Analysis on Rogowski-coil Current Transformer to Protective Relaying}

The unsaturation characteristics of Rogowski-coil Current Transformer have provided a solid foundation for its application in protective relaying system. But to replace electromagnetic current transformers not only requires the products have a very good maturity and stability, but also needs further improvement of Rogowski-coil Current Transformer in its performance, standards, and initiative in adapting to the demands of protective relaying.

1) The frequency characteristics of Rogowski-coil Current Transformer have a great effect on transient characteristics. So far the requirements of protective electronic current transformer on the accuracy of harmonics still follows the conventional standards of electromagnetic current transformers, which only consider the transfer accuracy of the harmonic within the 5th harmonic.

This paper believes that the requirements of Rogowskicoil current transformer on harmonic accuracy should not be limited to the harmonic times that protection principles and algorithms apply and should analyse the harmonic features of fault current or the magnetizing inrush current of transformer to determine the bandwidth of Rogowski-coil Current Transformer. To this end, the simulation for transformer no-load closing have been performed in RTDS, the magnetizing inrush current waveforms are shown in Figure 6. The harmonic analysis results show that the harmonic ratio of 11 th harmonic is up to $2.14 \%$. It indicates that the harmonics accuracy of transformers should at least take harmonics higher than 10th harmonics into account.

2) The lower-cut-off frequency of the band of Rogowski-coil Current Transformer is mainly limited by the integral unit parameters, and the upper cutoff frequency is mainly confined to the resonant frequency of the sensor, as shown in Figure 3.

Generally the manufacturers do not measure the distributed capacitance of the Rogowski coil; it is recommended that the resonance method should be used for measuring capacitance. And the specific bandwidth parameter of Rogowski-coil Current Transformer should be provided, which can provide reference for the selection of protective relaying applied.

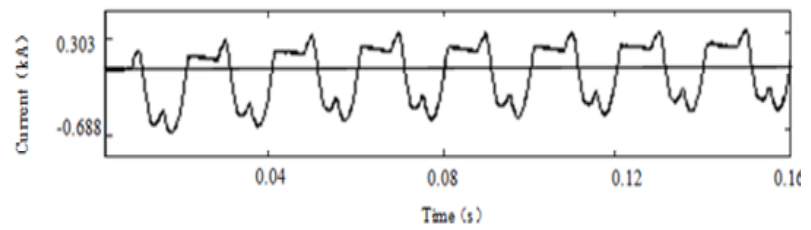

Figure 6. The excitation inrush current waveform from RTDS simulation.
3) The transient characteristics of Rogowski-coil Current Transformer can be quantitatively analyzed. If the frequency band is wide enough, the transient characteristics can be described by the ability to transfer decaying aperiodic component. Thus the design of the integrator for the differential signal of Rogowski transducer is particularly crucial.

This paper proposes that the existing test standards for Rogowski-coil Current Transformer can quantify the transfer characteristics of decaying a periodic component, and have no need to follow the transient standard for electromagnetic current transformer.

It is recommended that the test of transient characteristics can be performed by directly applying decaying dc. Considering the balance of the speed requirements of protective relaying and acceptable measurement errors, the transient errors should be limited to below a certain value (such as 5\%) in $40 \mathrm{~ms}$ after the failures occur.

4 ) Redundant configuration is widely used in the design and application of Rogowski-coil Current Transformer, such as double $\mathrm{A} / \mathrm{D}$ design in sampling unit, double configurations for transformers and merging units, two independent synchronization sources, two power standby and so on. Redundant configuration is an effective measure if the hardware or software of devices fail, and the technical difficulty lies in achieving a high degree of coordination of double circuit.

The switch between the external synchronization and the internal synchronization mode in a merging unit device has been tested, the test results are as shown in Figure 7 below. It can be found that when the synchronization mode of merging unit switches, a part of the data of the earlier cycle were resent and resulted in the distortion of output. Thus, transformer design should ensure effective stand-by and seamless switch; otherwise it may cause the decline of the protection reliability. It is necessary to analyze the most reasonable redundant configuration in the design of protective relaying system.

\section{Conclusions}

Rogowski-coil Current Transformer has the characteristics of a linear system, it will not saturate and have a

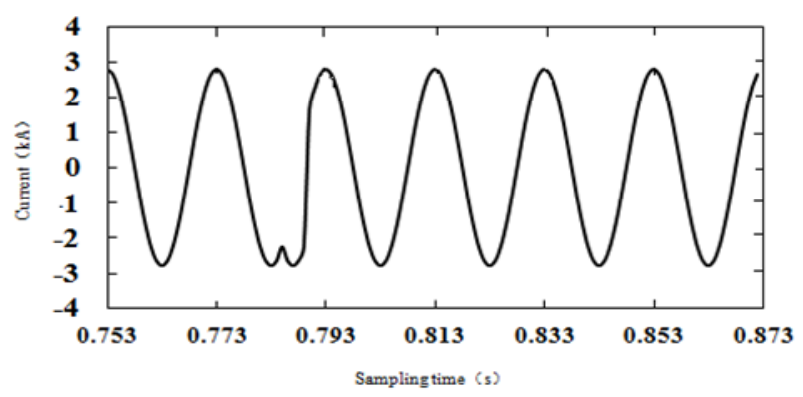

Figure 7. Synchronous mode switch of Merging unit. 
good linearity. Internal resistance and distributed capacitance of the sensor has an impact on its high-frequency characteristics, the high-frequency characteristic of the overall transformer is greatly affected by electronic circuits, especially the phase compensation circuit, other than limited by the sensor.

The errors of Rogowski-coil Current Transformer transferring the transient currents largely depend on whether the integrator can accurately restore the differential signal of the sensor or not. This research suggests that first-order RC integrator cannot meet the protective relaying requirements on transient characteristics of transformer. Following research on appropriate way of integration will be performed facing the protective relaying application.

This paper put forward some proposals on structure design and testing standards of the Rogowski-coil Current Transformer to improve transfer performance of transformers, so as to better adapt to application requirements of protective relaying.

\section{REFERENCES}

[1] D. A. Ward and J. La T. Exon, "Using Rogowski Coils for Transient Current Measurements," Engineering Science and Education Journal, Vol. 2, No. 3, 1993, pp. 105-113. doi:10.1049/esej:19930034

[2] L. Kojovic, "Rogowski Coils Suit Protective Relaying and Measurement," IEEE Computer Application in Power, Vol. 10, No. 2, 1997, pp. 47-52. doi:10.1109/67.595293

[3] J. D. Ramboz, "Machinable Rogowski Coil Design and Calibration," IEEE Transactions on Instrumentation and Measurement, Vol. 45, No. 2, 1996, pp. 511-515. doi:10.1109/19.492777

[4] S. N. Luo, Z. B. Tian and X. C. Zhao, "Performance analysis of Rogowski-coil Current Transformer," Proceedings of the CSEE, Vol. 24, No. 3, 2004, pp. 108-113.

[5] Q. Chen, H. B. Li, M. M.Zhang, et al., "Design and CharActeristics of Two Rogowski Coils Based on Printed Circuit Board," IEEE Transaction on Instrumentation and Measurement, Vol. 55, No. 3,2006, pp. 939-943. doi:10.1109/TIM.2006.873788

[6] C. Y. Wang, Y. P. Chen, G. Zhang, et al., "Analysis and Control on the Position Errors of PCB Air-core Coils,"
Proceedings of the CSEE, Vol. 55, No. 3, 2008, pp. 103-108.

[7] E. F. Donaldson, J. R. Gibson, G. R. Jones, et al.," Hybrid Optical Current Transformer with Optical and Power-line Energisation," IEE Proceedings-Generation Transmission and Distribution, Vol. 147, No. 5, 2000, pp. 304-309. doi:10.1049/ip-gtd:20000604

[8] G. Yang and X. R. Liu, "Improvements on Circuit Design for Highvoltage Side of Active Power Electronic Current Transducer," Power System Technology, Vol. 29, No. 14, 2005, pp. 81-84.

[9] K. W. Zhang, N. Wang, X. Y. Duan, et al., "A Digital Integrator for Electronic Current Transducer," Proceedings of the CSEE, Vol. 24, No. 12, 2004, pp. 104-107.

[10] Y. Wang, B. d. Cao and T. Jiang, "Fast Data Processing for Merging Unit of Electronic Transformer," Power System Technology, Vol. 33, No. 1, 2009, pp. 87-91.

[11] W.B. Li, C.X. Mao, J.M. Lu, et al., "Study of the Influence of the Distributed Capacitance on Dynamic Property of Rogowski Coil," Transactions of China Electrotechnical Society, Vol. 19, No. 6, 2004, pp. 12-17.

[12] Y. Xu and B. Ran, "Analysis and Simulation of Air-core Coil Transducer's Response to Transient Current," Transactions of China Electrotechnical Society, Vol. 19, No. 11, 2004, pp. 61-65.

[13] G. Zhang, C.Y. Wang and Y. P. Chen, "Transient Behavior of PCB Air-core Coil Current Transformer," Transactions of China Electrotechnical Society, Vol. 25, No. 11, 2010, pp. 25-29.

[14] T. Wu, Y. Q. Zhou and W. Gong, "Performance Analysis on Electronic Current Transformer Based on PCB Planar-type of Air Core Coil," Power System Technology, Vol. 34, No. 6, 2010, pp. 210-214.

[15] IEC60044-8, "Instrument Transformers: Part 8 Electronic Current Transformers," 2002.

[16] GB/T20840.8-2007, "Instrument Transformers -Part:8: Electronic Current Transformers," 2007.

[17] J. Yu, X. F. Xiong and Y. Zhang, "Research and Simulation on New Reliability Measures for Digital Substation Protection System," Power System Technology, Vol. 33, No. 4, 2009, pp. 28-33.

[18] Z. Q. Li, Z.X. Zhou, Y. Huang, et al., "Research on Applicability of Protective Relaying in Digital Substations". Power System Technology, Vol. 35, No. 5, 2011, pp. 210-215. 